Article

\title{
Spinasterol, 22,23-Dihydrospinasterol and Fernenol from Citrullus Colocynthis L. with Aphicidal Activity against Cabbage Aphid Brevicoryne Brassicae L.
}

\author{
Maqsood Ahmed ${ }^{1,2}{ }^{-}$, Peiwen Qin ${ }^{1, *}$, Mingshan Ji ${ }^{1, *}$, Ran An ${ }^{1}$, Hongxia Guo ${ }^{1}$ and \\ Jamil Shafi ${ }^{3}$ (D) \\ 1 College of Plant Protection, Shenyang Agricultural University, Shenyang 110866, China; \\ maqsoodahmed200@hotmail.com (M.A.); anan8u8@outlook.com (R.A.); ghx331682817@163.com (H.G.) \\ 2 Department of Agriculture, Pest Warning \& Quality Control of Pesticides, Gujrat 50700, Pakistan \\ 3 Department of Plant Pathology, University of Agriculture Faisalabad, Sub-Campus Depalpur, Okara, \\ Faisalabad 56300, Pakistan; jamil_shafi786@yahoo.com \\ * Correspondence: qinpeiwen@syau.edu.cn (P.Q.); jimingshan@syau.edu.cn (M.J.); \\ Tel.: +86-24-8848-7148 (M.J.); Fax: +86-24-8834-2315 (M.J.)
}

Academic Editor: Giovanni Benelli

Received: 7 April 2020; Accepted: 29 April 2020; Published: 7 May 2020

\begin{abstract}
Brevicoryne brassicae is a problematic pest in cabbage and other field crops. Synthetic pesticides are used to control this pest, but they are injurious for human health and the environment. The present study aimed to purify and identify the active compounds from Citrullus colocynthis leaves with an appraisal of their efficacy against B. brassicae. Separation and purification were performed via different chromatographic techniques. Molecular analysis and chemical structures were recognized by mass spectrum (MS) and nuclear magnetic resonance (NMR), respectively. Moreover, in vitro and in vivo aphicidal activity was assessed using various concentrations, i.e., 6.25, 12.5, 25 and $50 \mu \mathrm{g} / \mathrm{mL}$ at 12, 24, 48 and $72 \mathrm{~h}$ exposure. The outcome shows that mass spectrum analyses of the purified compounds suggested the molecular formulae are $\mathrm{C}_{30} \mathrm{H}_{50} \mathrm{O}$ and $\mathrm{C}_{29} \mathrm{H}_{50} \mathrm{O}, \mathrm{C}_{29} \mathrm{H}_{48} \mathrm{O}$. The compounds were characterized as fernenol and a mixture of spinasterol, 22,23-dihydrospinasterol by ${ }^{1} \mathrm{H}-\mathrm{NMR}$ and ${ }^{13} \mathrm{C}$-NMR spectrum analysis. The toxicity results showed that the mixture of spinasterol and 22,23-dihydrospinasterol showed $\mathrm{LC}_{50}$ values of $32.36,44.49$ and $37.50 \mu \mathrm{g} / \mathrm{mL}$ by contact, residual and greenhouse assay at $72 \mathrm{~h}$ exposure, respectively. In contrast, fernenol recorded $\mathrm{LC}_{50}$ values as $47.99,57.46$ and $58.67 \mu \mathrm{g} / \mathrm{mL}$, respectively. On the other hand, spinasterol, 22,23-dihydrospinasterol showed the highest mortality, i.e., $66.67 \%, 53.33 \%$ and $60 \%$ while, $30 \%, 23.33 \%$ and $25 \%$ mortality was recorded by fernenol after $72 \mathrm{~h}$ at $50 \mu \mathrm{g} / \mathrm{mL}$ by contact, residual and greenhouse assay, respectively. This study suggests that spinasterol, 22,23-dihydrospinasterol are more effective against $B$. brassicae which may be introduced as an effective and suitable substitute of synthetic chemical pesticides.
\end{abstract}

Keywords: Citrullus colocynthis; spinasterol, 22,23-dihydrospinasterol; fernenol; insecticidal activity; $\mathrm{LC}_{50}$

\section{Introduction}

The cabbage aphid, Brevicoryne brassicae L. belongs to (Hemiptera: Aphididae) is one of the key pest of vegetables and crops, commonly distributed in warm and moderate areas of the world [1,2]. Due to the heavy infestation of B. brassicae on cabbage, yield losses can be increased up to $70 \%$ and under-promising circumstances, it can cause complete loss of leafy vegetables of brassica [3,4]. Being an injurious pest, it negatively affects cabbage production by developing sooty mold on plants surface $[2,5]$. Continued feeding of this pest caused yellowing, wilting and stunting of plant growth, ultimately 
cause plant death and lead to economic losses [6]. Moreover, most of the species of aphid have gained resistance against many synthetic aphicidal agents [7].

Although synthetic chemicals are widely used to manage this pest but, intensive and continuous use of these chemicals has resulted in the development of pest resistance, resurgence to these chemicals and may also leave hazardous effects on humans and the environment [8]. In contrast, botanical insecticides are relatively safe to some extent and are effective substitutes of these chemicals which play a fundamental role in the field of biopesticides. However, plant derivatives play a vital role in their biologic tasks [9]. Numerous findings have specified that phenols, terpenoids and nitrogen-based constituents are imperative phytoalexins which afford a protective system to plants being attacked by other injurious insect-pests $[9,10]$.

Citrullus colocynthis is an important plant from the pharmacological and pesticidal viewpoint. It belongs to the Cucurbitaceae family, mostly grows in desert areas and has attracted the consideration of researchers as a natural botanic pesticide. The insecticidal potential of this desert plant has been assessed against various insect species [11]. Citrullus colocynthis exerts carcinogenic, antidiabetic, antibacterial, antioxidant properties, and possesses toxic properties against several harmful insects [12-15]. Numerous active chemical compounds have been reported from $C$. colocynthis, including bitter materials (colocynthetin and colocynthin), various cucurbitacins such as A, B, C, D and E [16], other cucurbitacins like E, I, J, K and L [17], cucurbitacins glycosides [18,19], the cucurbitacins glucosides I and L [19], flavone glycosides and flavonoids $[19,20]$. However, the cucurbitacins tetracyclic triterpens possess extensive biologic activities. Numerous biologic compounds belong to this group being examined because of their cytotoxic, hepatoprotective, cardiovascular, antioxidant action of B and I cucurbitacins [21].

Song et al. [22] isolated two cucurbitacins from the ethyl acetate extract of $C$. colocynthis fruit, but these were not evaluated against insects. Similarly, Ding et al. [23] identified a mixture of spinasterol, 22,23-dihydrospinasterol from the roots of Bermeuxia thibetica, but its bioactivity was also not evaluated. However, Sinha et al. [24] reported that spinasterol, 22,23-dihydrospinasterol exhibited by Melothria maderaspatana showed biologic activities. It has been reported that Artemisia extracts contain valuable phytochemicals, possess insecticidal activity which were mainly attributed to the presence of fernenol and other phytoconstituents [25]. Furthermore, constituents of Artemisia vulgaris like psilostachy C, Maackiain, psilostachyin A and fernenol possess medicinal as well as antibacterial activities and also used by farmers for the preservation of crops and stored grains products [26].

Although some studies have been conducted on isolation and identification of various compounds including spinasterol, 22,23-dihydrospinasterol and fernenol from natural plant resources, their use as insecticidal purposes especially against B. brassicae has not been evaluated so far. Therefore, the present novel study was conducted in a comprehensive way for the isolation, characterization and evaluation of biochemical compounds from C. colocynthis leaves against B. brassicae.

\section{Results}

\subsection{Preliminary Toxicity Evaluations}

Interestingly, all solvents extract caused significant mortality of B. brassicae. However, maximum mortality $(85 \%)$ was recorded at $96 \mathrm{~h}$ post exposure by methanol extract, followed by ethanol and chloroform extract $(80 \%)$ and $(76.67 \%)$, respectively. In contrast, at $72 \mathrm{~h}$ exposure, maximum mortality afforded by methanol extract was $66.67 \%$, followed by chloroform and ethanol extract $(58.33 \%$ ) and $(53.33 \%)$, respectively. However, minimum mortality was recorded via distilled water extract which was $35 \%$ and $18.33 \%$ at 96 and $72 \mathrm{~h}$ exposure, respectively. The results also revealed that mortality is dependent upon concentration and prolonged time exposure (Figure 1). 


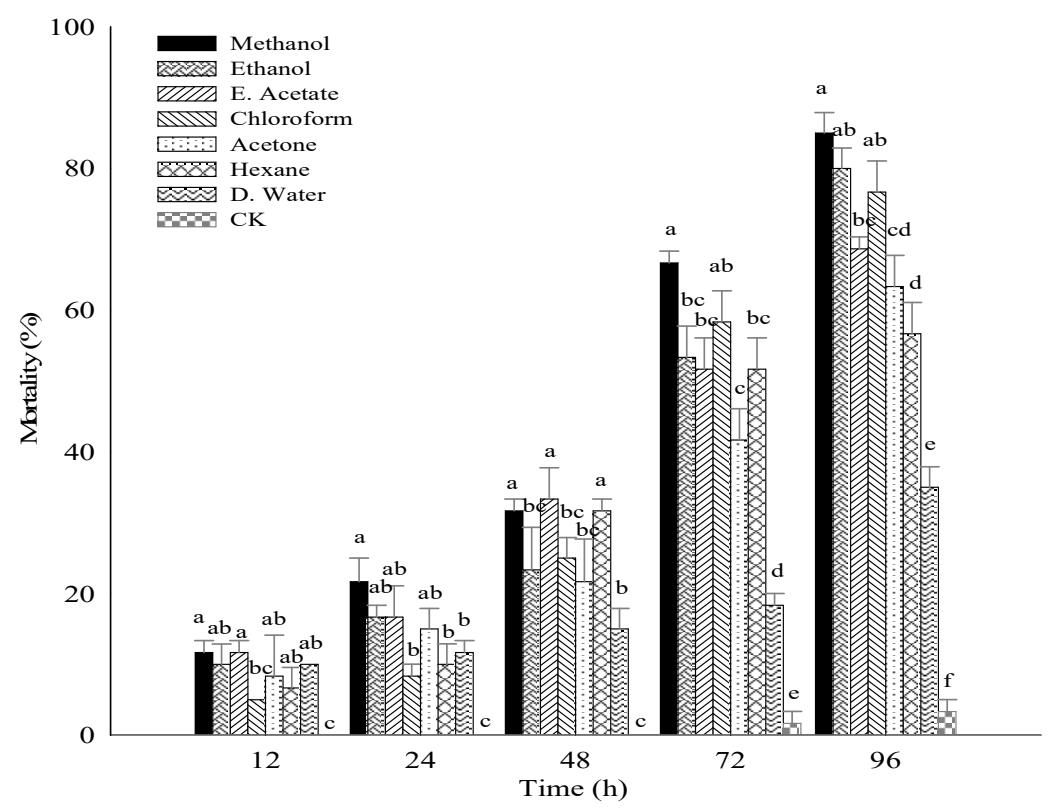

Figure 1. Mortality of Brevicoryne brassicae by Citrullus colocynthis leaves extracts. Values are represented as mean \pm standard deviation followed by different superscripts $(a, b, c, d, e, f, a b, b c$, cd) were not significantly different according to Duncan multiple range test (DMRT) at $p>0.05$. E. Acetate (ethyl acetate); D. Water (distilled water); CK (check in distilled water); Time (h).

\subsection{Extraction, Separation and Purification of Extract}

Powdered leaves of $C$. colocynthis were extracted with different polarity based solvents. Fractionation of the extract was performed by chromatographic techniques. Collected fractions followed by separation by silica gel column were concentrated and weighed following their Thin Layer Chromatography (TLC) analysis (Table 1). Out of 8 of the obtained fractions, $\mathrm{D}_{2}$ fraction was further purified by repeated column chromatography on silica gel column, Sephadex gel column and preparative TLC and finally two compounds referred to as D2 (N) and D3A bearing weight $35 \mathrm{mg}$ and $21 \mathrm{mg}$, respectively were purified.

Table 1. Obtained fractions by silica gel column.

\begin{tabular}{ccc}
\hline Eluted Fractions & Collected Fractions & Weight $(\mathrm{g})$ \\
\hline $1-10$ & A & 0.293 \\
$11-13$ & B & 0.355 \\
$14-23$ & C & 0.796 \\
$24-27$ & D & 0.662 \\
$28-30$ & E & 0.710 \\
$31-34$ & F & 0.270 \\
$35-40$ & G & 0.447 \\
$41-43$ & H & 0.428 \\
$44-49$ & I & 2.841 \\
\hline
\end{tabular}

\subsection{Mass Spectrum and Elemental Analysis}

The $\mathrm{C}, \mathrm{H}$ and $\mathrm{N}$ elemental analysis is helpful for the calculation of empirical formula. The mass spectrometer examination recorded the molecular ion peak $(\mathrm{M})$ at $m / z 426,414$ and 412 . These data suggested the expected molecular formula as $\mathrm{C}_{30} \mathrm{H}_{50} \mathrm{O}$ (Figure 2) and a mixture of $\mathrm{C}_{29} \mathrm{H}_{50} \mathrm{O}, \mathrm{C}_{29} \mathrm{H}_{48} \mathrm{O}$ (Figure 3) for D3A and D2 (N), respectively. However, D2 $(\mathrm{N})$ was a mixture of two compounds referred to as D2 (N1) and D2 (N2) and the ratio of these two compounds was recorded as 5:4 from the mass spectrum and nuclear magnetic resonance analysis. 


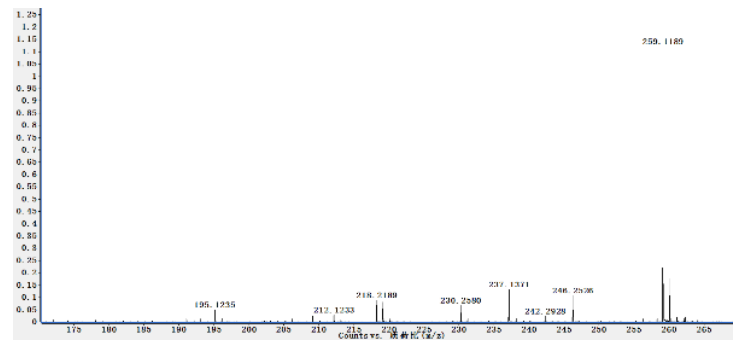

(a)

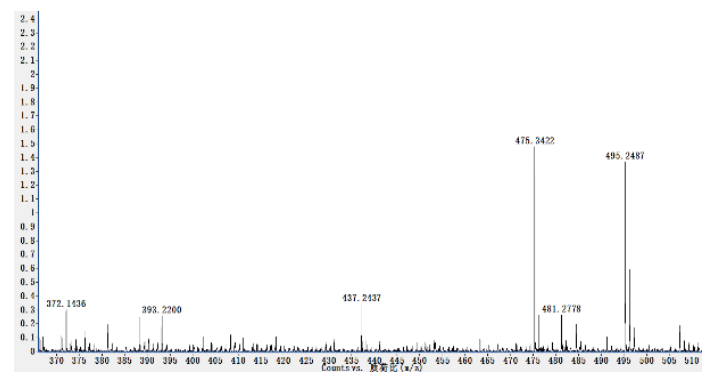

(c)

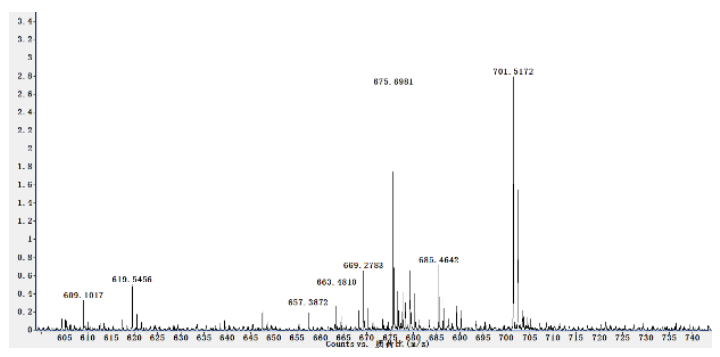

(e)

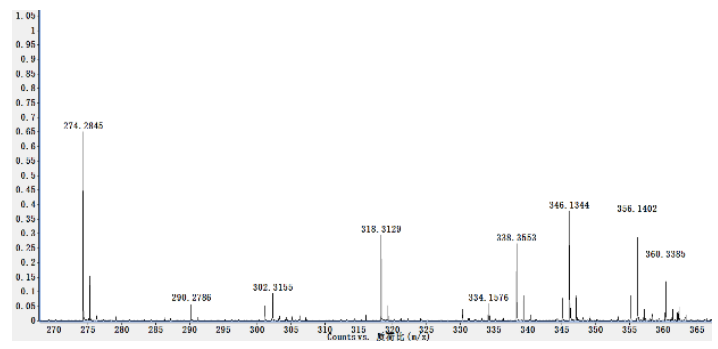

(b)

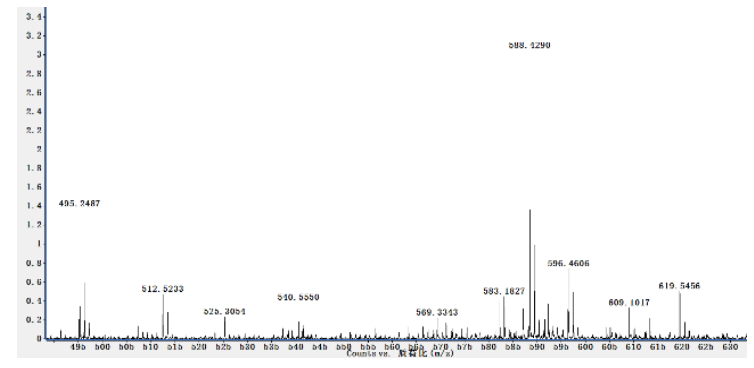

(d)

Figure 2. (a-e) The full mass spectrum for the purified compound, D3A from Citrullus colocynthis leaves.

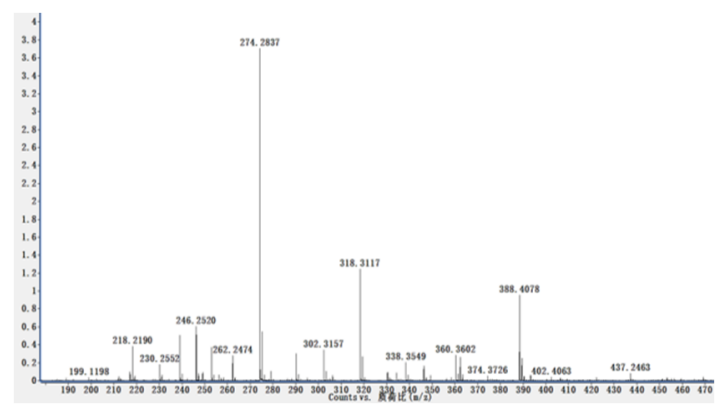

(a)

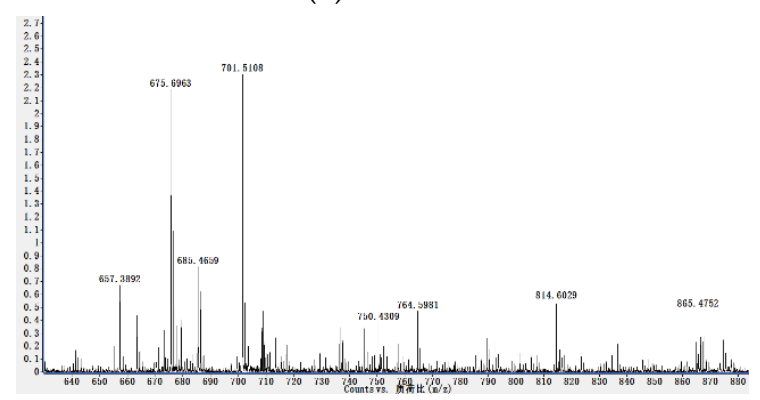

(c)

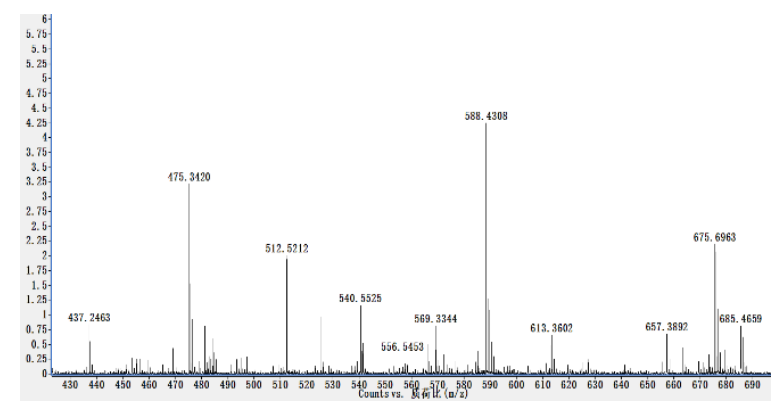

(b)

Figure 3. (a-c) The full mass spectrum for the purified compound, D2 (N) from Citrullus colocynthis leaves. 


\subsection{NMR Analysis $\left({ }^{1} H-N M R\right.$ and $\left.{ }^{13} \mathrm{C}-N M R\right)$}

The chemical structures of the purified compounds D2 $(\mathrm{N})$ and D3A were characterized by nuclear magnetic resonance (NMR), Bruker BioSpin, Billerica MA for $1 \mathrm{D}\left({ }^{1} \mathrm{H}-\mathrm{NMR},{ }^{13} \mathrm{C}-\mathrm{NMR}\right)$, mass spectrum and elemental analysis. All NMR experiments were carried out at room temperature.

In the ${ }^{1} \mathrm{H}-\mathrm{NMR}$ and ${ }^{13} \mathrm{C}-\mathrm{NMR}$ spectrum of the compound $\mathrm{D}_{2}(\mathrm{~N} 1)^{1} \mathrm{H}-\mathrm{NMR}\left(600 \mathrm{MHz}, \mathrm{CDCl}_{3}\right) \delta$ $5.16(\mathrm{~m}, 1 \mathrm{H}, \mathrm{H}-7), 3.60(\mathrm{~m}, 1 \mathrm{H}, \mathrm{H}-3)$ allylic protons, $0.93\left(\mathrm{~d}, J=7.0 \mathrm{~Hz}, 3 \mathrm{H},-\mathrm{CH}_{3}\right), 0.85(\mathrm{t}, J=6.8 \mathrm{~Hz}$, $\left.3 \mathrm{H},-\mathrm{CH}_{3}\right), 0.82\left(\mathrm{~d}, J=6.9 \mathrm{~Hz}, 3 \mathrm{H},-\mathrm{CH}_{3}\right), 0.81\left(\mathrm{~d}, J=6.9 \mathrm{~Hz}, 3 \mathrm{H},-\mathrm{CH}_{3}\right), 0.80\left(\mathrm{~s}, 3 \mathrm{H},-\mathrm{CH}_{3}\right), 0.55(\mathrm{~s}$, $3 \mathrm{H},-\mathrm{CH}_{3}$ ) (Figure 4a). ${ }^{13} \mathrm{C}-\mathrm{NMR}\left(151 \mathrm{MHz}, \mathrm{CDCl}_{3}\right) \delta 11.8,12.0,13.0,18.8,19.0,19.7,21.5,22.9,23.0$, 26.1, 27.9, 29.1, 29.6, 31.4, 33.8, 34.1, 36.5, 37.1,37.9, 39.5, 40.2, 43.3, 45.8, 49.4, 55.0, 56.0, 71.0, 117.3, 139.5, (Spinasterol, $\mathrm{C}_{29} \mathrm{H}_{50} \mathrm{O} ; 414.0 \mathrm{~g} / \mathrm{mol}$ ) (Figure $4 \mathrm{~b}$ ). However, ${ }^{1} \mathrm{H}-\mathrm{NMR}$ and ${ }^{13} \mathrm{C}-\mathrm{NMR}$ spectra of the compound $\mathrm{D}_{2}(\mathrm{~N} 2){ }^{1} \mathrm{H}-\mathrm{NMR}\left(600 \mathrm{MHz}, \mathrm{CDCl}_{3}\right) \delta 5.16$ (overlap, $\left.1 \mathrm{H} \mathrm{H}-7\right), 5.16$ (overlap, $1 \mathrm{H}, \mathrm{H}-22$ ), $5.03(\mathrm{dd}, J=15.1,8.8 \mathrm{~Hz}, 1 \mathrm{H}, \mathrm{H}-23), 3.60(\mathrm{~m}, 1 \mathrm{H}, \mathrm{H}-3)$, ) the allylic protons, $1.03(\mathrm{~d}, J=7.0 \mathrm{~Hz}, 3 \mathrm{H}$, $\left.-\mathrm{CH}_{3}\right), 0.85\left(\mathrm{t}, J=6.8 \mathrm{~Hz}, 3 \mathrm{H},-\mathrm{CH}_{3}\right), 0.82\left(\mathrm{~d}, J=6.9 \mathrm{~Hz}, 3 \mathrm{H},-\mathrm{CH}_{3}\right), 0.81\left(\mathrm{~d}, J=6.9 \mathrm{~Hz}, 3 \mathrm{H},-\mathrm{CH}_{3}\right), 0.80(\mathrm{~s}$, $\left.3 \mathrm{H},-\mathrm{CH}_{3}\right), 0.54\left(\mathrm{~s}, 3 \mathrm{H},-\mathrm{CH}_{3}\right)$ (Figure 4). ${ }^{13} \mathrm{C}-\mathrm{NMR}\left(151 \mathrm{MHz}, \mathrm{CDCl}_{3}\right) \delta 11.9,12.2,13.0,18.9,21.0,21.3$, 21.5, 22.9, 25.3, 28.4, 29.6, 31.4, 31.8, 34.1, 37.1, 37.9, 39.4, 40.2, 40.8, 43.2, 49.4, 51.2, 55.0, 55.8, 71.0, 117.4, 129.4, 138.1, 139.5, (22,23-dihydrospinasterol, $\mathrm{C}_{29} \mathrm{H}_{48} \mathrm{O} ; 412.0 \mathrm{~g} / \mathrm{mol}$ ) (Figure $4 \mathrm{~b}$ ).

In the ${ }^{1} \mathrm{H}-\mathrm{NMR}$ and ${ }^{13} \mathrm{C}-\mathrm{NMR}$ spectra of the compound $\mathrm{D}_{3} \mathrm{~A},{ }^{1} \mathrm{H}-\mathrm{NMR}\left(600 \mathrm{MHz}, \mathrm{CDCL}_{3}\right) \delta 5.30$ $(\mathrm{s}, 1 \mathrm{H}, \mathrm{C}=\mathrm{CH}), 3.26-3.17(\mathrm{~m}, 1 \mathrm{H},-\mathrm{OH})$, the allylic protons, $1.07\left(\mathrm{~s}, 3 \mathrm{H},-\mathrm{CH}_{3}\right), 0.96\left(\mathrm{~s}, 3 \mathrm{H},-\mathrm{CH}_{3}\right), 0.89(\mathrm{~d}$, $\left.J=6.4 \mathrm{~Hz}, 3 \mathrm{H},-\mathrm{CH}_{3}\right), 0.87\left(\mathrm{~s}, 3 \mathrm{H},-\mathrm{CH}_{3}\right), 0.84-0.80\left(\mathrm{~m}, 6 \mathrm{H}, \mathrm{two}-\mathrm{CH}_{3}\right), 0.76\left(\mathrm{~s}, 3 \mathrm{H},-\mathrm{CH}_{3}\right), 0.73(\mathrm{~s}, 3 \mathrm{H}$, $\left.-\mathrm{CH}_{3}\right), 0.73-2.03\left(\mathrm{~m}, 48 \mathrm{H}\right.$, including $\left.8 \mathrm{CH}_{3}\right)$ (Figure 4c). ${ }^{13} \mathrm{C}-\mathrm{NMR}\left(151 \mathrm{MHz}, \mathrm{CDCL}_{3}\right) \delta: 13.90,15.77$, $17.87,19.07,20.05,22.04,22.98,25.13,27.37,28.13,28.06,29.20,29.63,30.69,36.07,36.65,37.57,37.70$, $39.19,39.27,39.90,40.96,42.85,44.20,50.29,51.88,59.58,79.07,116.11,150.98$, (Fernenol; $\mathrm{C}_{30} \mathrm{H}_{50} \mathrm{O}$; $426.7 \mathrm{~g} / \mathrm{mol}$ ) (Figure $4 \mathrm{~d}$ ).

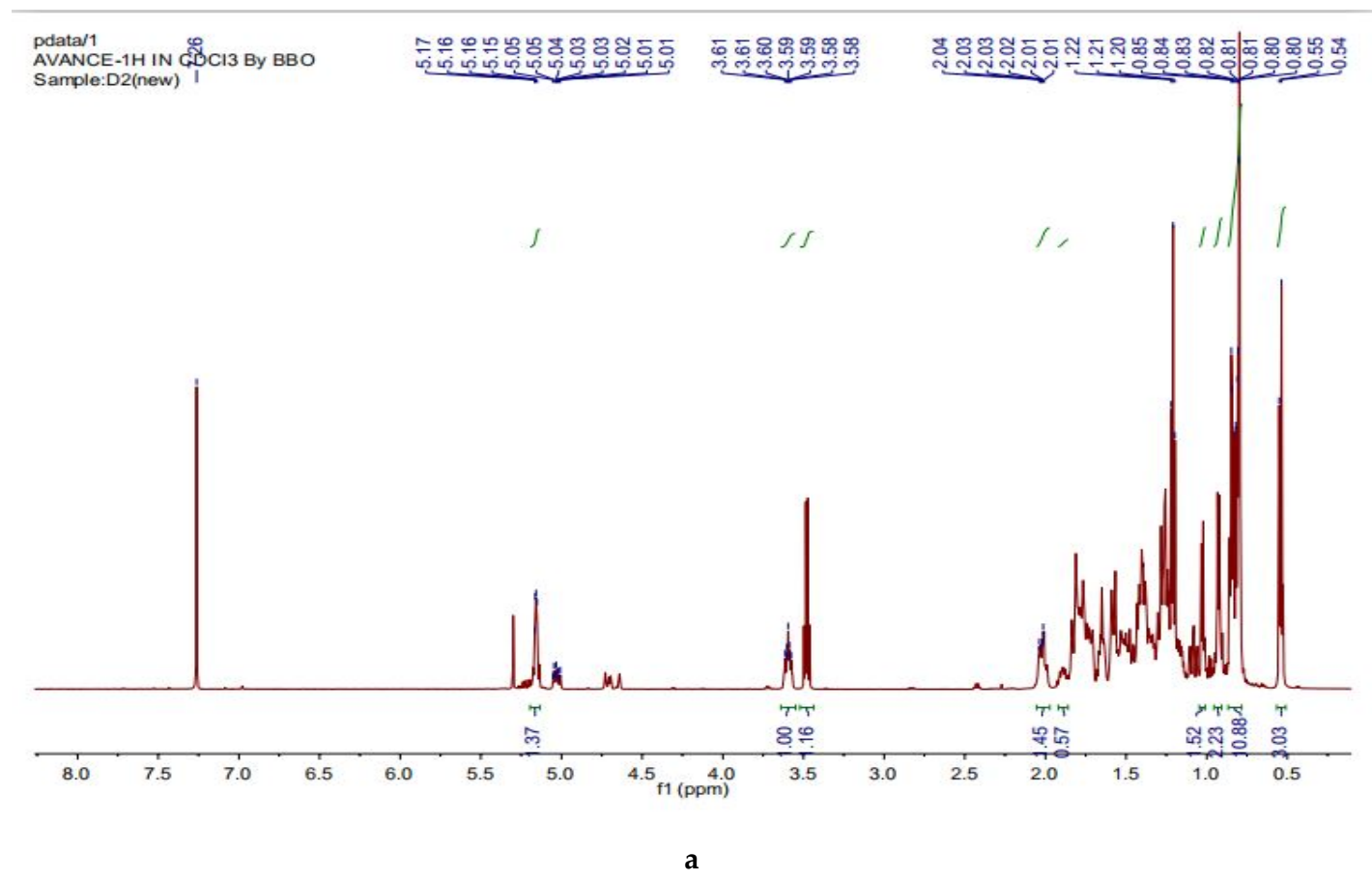

Figure 4. Cont. 


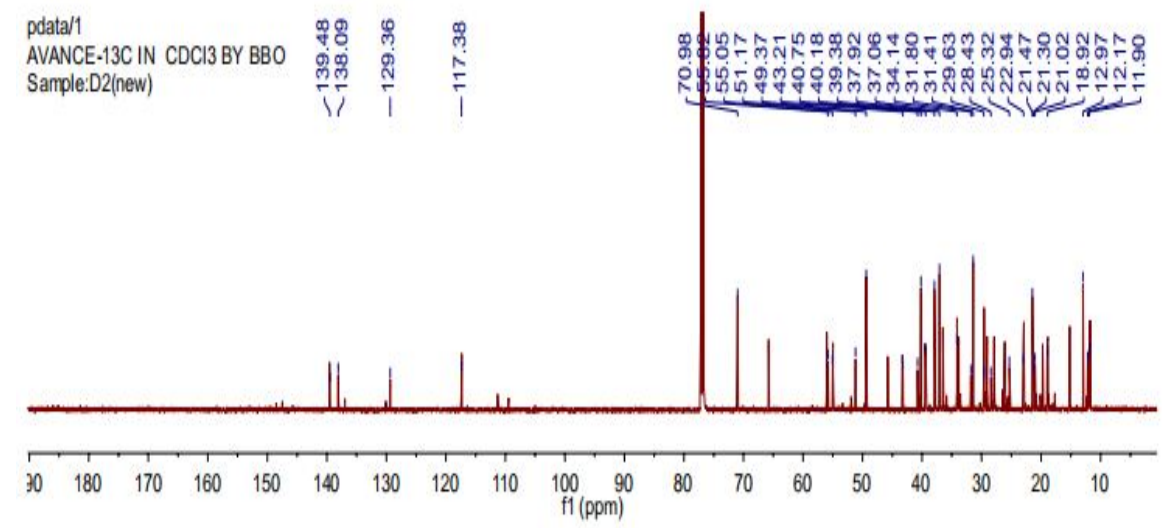

b
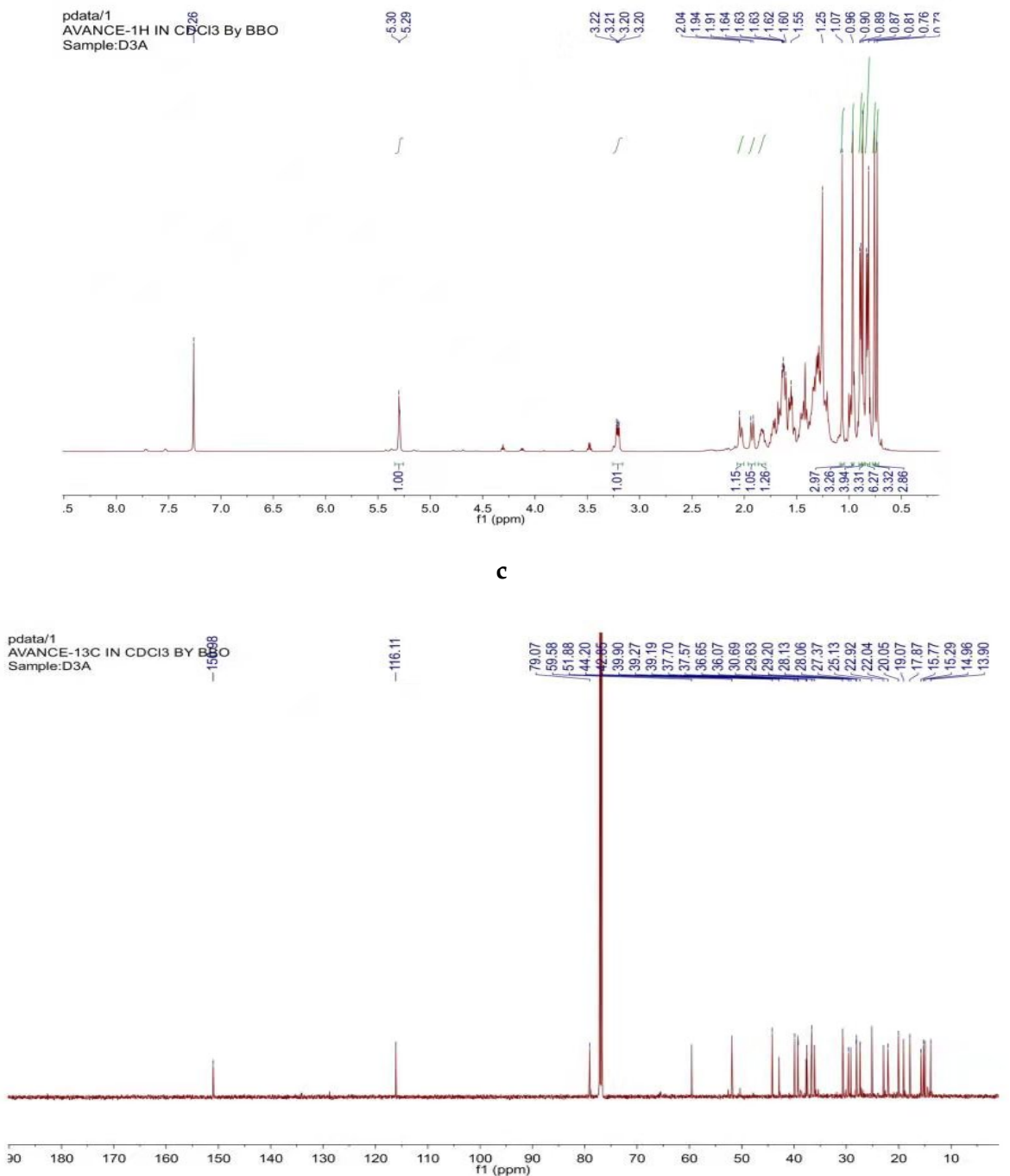

d

Figure 4. (a). ${ }^{1} \mathrm{H}-\mathrm{NMR}$ spectrum of the compound D2 (N); (b). ${ }^{13} \mathrm{C}-\mathrm{NMR}$ spectrum of compound D2 (N); (c). ${ }^{1} \mathrm{H}-\mathrm{NMR}$ spectrum of the compound D3A; (d). ${ }^{13} \mathrm{C}-\mathrm{NMR}$ spectrum of the compound D3A. 


\subsection{Structures Elucidation of the Compounds}

Chemical structures of the identified fractions of D3A and D2 $(\mathrm{N})$ were characterized by ${ }^{1} \mathrm{H}-\mathrm{NMR}$ and ${ }^{13} \mathrm{C}$-NMR spectroscopy and their molecular formulae were predicted by the mass spectrum. D3A compound was identified as fernenol (Figure 5). Another purified compound was a little bit impure and due to this impurity, the ${ }^{1} \mathrm{H}-\mathrm{NMR}$ spectrum was overlapped and hence, mixture of two compounds was identified as Spinasterol and 22, 23-dihydrospinasterol from the fraction D2 (N) with ratio 5:4 in the mixture (Figure 6a,b).

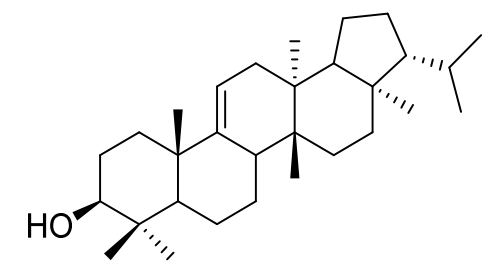

Figure 5. Chemical structure of compound; Fernenol from D3A.

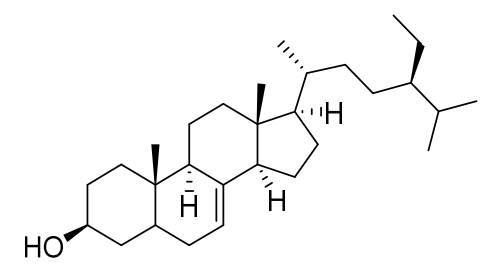

(a)

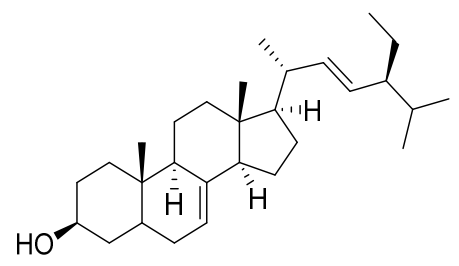

(b)

Figure 6. Chemical structures of compound; (a). Spinasterol; (b) 22, 23-dihydrospinasterol from D2 (N).

\subsection{Bioassay Study}

Toxicity of the identified compounds was evaluated extensively against B. brassicae by contact, residual and greenhouse assay and data were collected after a specific period of time exposure.

\subsubsection{In Vitro Contact Toxicity}

The mortality data described in Table 2 exposed the contact efficacy of the spinasterol, 22,23-dihydrospinasterol and fernenol against B. brassicae. It was observed that the percent mortality rate of aphids was directly associated with the concentration and period of exposure. Results indicated that extreme mortality recorded was $66.67 \%$ and $56.66 \%$ at 72 and 48 h exposure, respectively by spinasterol, 22,23-dihydrospinasterol at $50 \mu \mathrm{g} / \mathrm{mL}$. In contrast, moderate mortality was recorded by fernenol which was $30.00 \%$ and $21.67 \%$ at 72 and 48 h exposure, respectively, at the same concentration.

\subsubsection{In Vitro Residual Toxicity}

The mortality data described in Table 3 exposed the residual efficacy of spinasterol, 22,23-dihydrospinasterol and fernenol against B. brassicae. Results demonstrated that highest mortality (53.33\%) was observed at exposure of $72 \mathrm{~h}$ followed by $(43.33 \%)$ at $48 \mathrm{~h}$ by spinasterol, 22,23-dihydrospinasterol at the concentration of $50 \mu \mathrm{g} / \mathrm{mL}$. On the other hand, fernenol afforded lower mortality, i.e., $(23.33 \%)$ and (16.67\%) at the same exposure time and concentration, respectively.

\subsubsection{In Vivo Assay}

The mortality data presented in Table 4 revealed the insecticidal activity of the purified compounds against $B$. brassicae under greenhouse conditions. Results showed that mortality rate of aphids under greenhouse conditions was $60.00 \%$ at $72 \mathrm{~h}$ exposure via spinasterol, 22,23-dihydrospinasterol at $50 \mu \mathrm{g} / \mathrm{mL}$. In contrast, mortality recorded by fernenol was $25.00 \%$ at $50 \mu \mathrm{g} / \mathrm{mL}$ concentration and $72 \mathrm{~h}$ exposure. 
Table 2. Mortality of Brevicoryne brassicae by spinasterol, 22,23-dihydrospinasterol and fernenol via contact assay.

\begin{tabular}{|c|c|c|c|c|c|c|c|c|}
\hline \multirow{3}{*}{$\begin{array}{l}\text { Conc. } \\
(\mu \mathrm{g} / \mathrm{mL})\end{array}$} & \multicolumn{8}{|c|}{ Mean Mortality (\%) with Time (h) } \\
\hline & \multicolumn{2}{|l|}{12} & \multicolumn{2}{|l|}{24} & \multicolumn{2}{|l|}{48} & \multicolumn{2}{|l|}{72} \\
\hline & $\begin{array}{c}\text { Spinasterol, 22,23-Dihydr- } \\
\text { Spinasterol }\end{array}$ & Fernenol & $\begin{array}{c}\text { Spinasterol, 22,23-Dihydr- } \\
\text { Spinasterol }\end{array}$ & Fernenol & $\begin{array}{c}\text { Spinasterol, 22,23-Dihydr- } \\
\text { Spinasterol }\end{array}$ & Fernenol & $\begin{array}{c}\text { Spinasterol, 22,23-Dihydr- } \\
\text { Spinasterol }\end{array}$ & Fernenol \\
\hline 6.25 & $0.00 \pm 0.00^{c}$ & $0.00 \pm 0.00^{\mathrm{a}}$ & $0.00 \pm 0.00^{c}$ & $0.00 \pm 0.00^{c}$ & $3.33 \pm 3.33^{\mathrm{c}}$ & $1.67 \pm 1.67^{c}$ & $6.67 \pm 3.33^{c}$ & $3.33 \pm 1.67^{c}$ \\
\hline 12.50 & $3.33 \pm 3.33^{c}$ & $0.00 \pm 0.00^{\mathrm{a}}$ & $10.00 \pm 0.00^{c}$ & $5.00 \pm 2.87^{b c}$ & $13.3 \pm 3.33^{c}$ & $6.67 \pm 1.67^{\mathrm{bc}}$ & $20.00 \pm 5.77^{c}$ & $10.0 \pm 2.89^{b c}$ \\
\hline 25 & $16.67 \pm 3.33^{\mathrm{b}}$ & $3.33 \pm 1.67^{\mathrm{a}}$ & $23.33 \pm 3.33^{\mathrm{b}}$ & $8.33 \pm 1.67^{\mathrm{ab}}$ & $26.67 \pm 3.33^{b}$ & $11.67 \pm 1.67^{\mathrm{b}}$ & $43.33 \pm 6.67^{b}$ & $15.0 \pm 2.89^{b}$ \\
\hline 50 & $26.67 \pm 3.33^{\mathrm{a}}$ & $3.3 \pm 1.67^{\mathrm{a}}$ & $40.00 \pm 0.00^{\mathrm{a}}$ & $13.3 \pm 1.67^{\mathrm{a}}$ & $56.67 \pm 3.33^{\mathrm{a}}$ & $21.67 \pm 1.67^{\mathrm{a}}$ & $66.67 \pm 3.33^{\mathrm{a}}$ & $30.002 .89^{a}$ \\
\hline \multirow[t]{2}{*}{ CK } & $0.00 \pm 0.00^{c}$ & $0.00 \pm 0.00^{\mathrm{a}}$ & $0.00 \pm 0.00^{c}$ & $0.00 \pm 0.00^{c}$ & $3.33 \pm 3.33^{c}$ & $1.67 \pm 1.67^{c}$ & $3.33 \pm 3.33^{\text {ed }}$ & $3.33 \pm 1.67^{\mathrm{c}}$ \\
\hline & Statics Summary & & & & & & & \\
\hline S.S & 1693 & 40.00 & 3506 & 390.0 & 5960 & 840.0 & 8573 & 1460 \\
\hline $\mathrm{df}$ & 4 & 4 & 4 & 4 & 4 & 4 & 4 & 4 \\
\hline M.S & 423 & 10.00 & 876.6 & 97.50 & 1490 & 210.0 & 2143 & 365.0 \\
\hline $\mathrm{F}$ & $21.17^{* * *}$ & $3.00^{\mathrm{NS}}$ & $131.50^{* * *}$ & $11.70^{* * *}$ & $44.70^{* * *}$ & $25.20^{* * *}$ & $32.150^{* * *}$ & $19.91^{* * *}$ \\
\hline
\end{tabular}

Values are described as mean \pm standard error followed by different superscripts were significantly different according to (Duncan multiple range test DMRT $p>0.05)$. S.S (Sum of square); df (Degree of freedom); M.S (Mean square); F (Significance); CK (Check); NS (Non-significant); ${ }^{* * *}$ (Highly significant).

Table 3. Mortality of Brevicoryne brassicae by spinasterol, 22,23-dihydrospinasterol and fernenol via residual assay.

\begin{tabular}{|c|c|c|c|c|c|c|c|c|}
\hline \multirow{3}{*}{$\begin{array}{l}\text { Conc. } \\
(\mu \mathrm{g} / \mathrm{mL})\end{array}$} & \multicolumn{8}{|c|}{ Mean Mortality (\%) with Time (h) } \\
\hline & \multicolumn{2}{|l|}{12} & \multicolumn{2}{|l|}{24} & \multicolumn{2}{|l|}{48} & \multicolumn{2}{|l|}{72} \\
\hline & $\begin{array}{c}\text { Spinasterol, 22,23-Dihydro- } \\
\text { Spinasterol }\end{array}$ & Fernenol & $\begin{array}{l}\text { Spinasterol, 22,23-Dihydro- } \\
\text { Spinasterol }\end{array}$ & Fernenol & $\begin{array}{l}\text { Spinasterol, 22,23-Dihydro- } \\
\text { Spinasterol }\end{array}$ & Fernenol & $\begin{array}{c}\text { Spinasterol, 22,23-Dihydro- } \\
\text { Spinasterol }\end{array}$ & Fernenol \\
\hline 6.25 & $0.00 \pm 0.00^{\mathrm{b}}$ & $0.00 \pm 0.00^{\mathrm{a}}$ & $0.00 \pm 0.00^{c}$ & $0.00 \pm 0.00^{\mathrm{b}}$ & $3.33 \pm 3.33^{c}$ & $0.00 \pm 0.00^{c}$ & $6.67 \pm 3.33^{c}$ & $1.67 \pm 1.67^{c}$ \\
\hline 12.50 & $0.00 \pm 0.00^{\mathrm{b}}$ & $0.00 \pm 0.00^{\mathrm{a}}$ & $3.33 \pm 3.33^{c}$ & $1.67 \pm 1.67^{\mathrm{b}}$ & $6.67 \pm 3.33^{c}$ & $3.33 \pm 1.67^{\mathrm{bc}}$ & $13.33 \pm 3.33^{c}$ & $5.00 \pm 0.00^{\mathrm{bc}}$ \\
\hline 25 & $6.67 \pm 3.33^{b}$ & $1.67 \pm 1.67^{\mathrm{a}}$ & $13.33 \pm 3.33^{b}$ & $5.00 \pm 2.89^{\mathrm{b}}$ & $26.67 \pm 3.33^{\mathrm{b}}$ & $6.67 \pm 1.67^{b}$ & $36.676 \pm 3.33^{b}$ & $10.00 \pm 2.89^{\mathrm{b}}$ \\
\hline 50 & $13.33 \pm 3.33^{\mathrm{a}}$ & $1.67 \pm 1.67^{\mathrm{a}}$ & $23.33 \pm 3.33^{\mathrm{a}}$ & $11.67 \pm 1.67^{\mathrm{a}}$ & $43.33 \pm 3.33^{\mathrm{a}}$ & $16.67 \pm 1.67^{\mathrm{a}}$ & $53.33 \pm 3.33^{\mathrm{a}}$ & $23.33 \pm 1.67^{\mathrm{a}}$ \\
\hline \multirow[t]{2}{*}{ CK } & $0.00 \pm 0.00^{\mathrm{b}}$ & $0.00 \pm 0.00^{\mathrm{a}}$ & $0.00 \pm 0.00^{c}$ & $0.00 \pm 0.00^{\mathrm{b}}$ & $0.00 \pm 0.00^{c}$ & $3.33 \pm 1.67^{\mathrm{bc}}$ & $3.33 \pm 3.33^{c}$ & $3.33 \pm 3.33^{c}$ \\
\hline & Statics summary & & & & & & & \\
\hline S.S & 426.6 & 10.00 & 1240 & 290.0 & 4093 & 493.3 & 5560.0 & 923.3 \\
\hline $\mathrm{df}$ & 4 & 4 & 4 & 4 & 4 & 4 & 4 & 4 \\
\hline M.S & 106.67 & 2.50 & 310.0 & 72.50 & 1023 & 123.3 & 1390 & 230.8 \\
\hline $\mathrm{F}$ & $8.00^{* *}$ & $0.750^{*}$ & $15.00^{* * *}$ & $8.70^{* * *}$ & $38.37^{* * *}$ & $18.50^{* * *}$ & $41.70^{* * *}$ & $23.08^{* * *}$ \\
\hline
\end{tabular}

Values are described as mean \pm standard error followed by different superscripts were significantly different according to (Duncan multiple range test DMRT $p>0.05$ ). S.S (Sum of square); df (Degree of freedom); M.S (Mean square); F (Significance); CK (Check); *** (Highly significant); ** (Highly significant); * (Significant). 
Table 4. Mortality of Brevicoryne brassicae by spinasterol, 22,23-dihydrospinasterol and fernenol via greenhouse assay.

\begin{tabular}{|c|c|c|c|c|c|c|c|c|}
\hline \multirow{3}{*}{$\begin{array}{l}\text { Conc. } \\
(\mu \mathrm{g} / \mathrm{mL})\end{array}$} & \multicolumn{8}{|c|}{ Mean Mortality (\%) with Time (h) } \\
\hline & \multicolumn{2}{|l|}{12} & \multicolumn{2}{|l|}{24} & \multicolumn{2}{|l|}{48} & \multicolumn{2}{|l|}{72} \\
\hline & $\begin{array}{l}\text { Spinasterol, 22,23-Dihydro- } \\
\text { Spinasterol }\end{array}$ & Fernenol & $\begin{array}{l}\text { Spinasterol, 22,23-Dihydro- } \\
\text { Spinasterol }\end{array}$ & Fernenol & $\begin{array}{c}\text { Spinasterol, 22,23-Dihydro- } \\
\text { Spinasterol }\end{array}$ & Fernenol & $\begin{array}{c}\text { Spinasterol, 22,23-Dihydro- } \\
\text { Spinasterol }\end{array}$ & Fernenol \\
\hline 6.25 & $0.00 \pm 0.00^{\mathrm{b}}$ & $0.00 \pm 0.00^{\mathrm{a}}$ & $0.00 \pm 0.00^{\mathrm{b}}$ & $0.00 \pm 0.00^{c}$ & $0.00 \pm 0.00^{c}$ & $0.00 \pm 0.00^{\mathrm{d}}$ & $3.33 \pm 3.33^{\mathrm{cd}}$ & $5.00 \pm 2.89^{c}$ \\
\hline 12.50 & $3.33 \pm 3.33^{b}$ & $0.00 \pm 0.00^{\mathrm{a}}$ & $6.67 \pm 3.33^{b}$ & $3.33 \pm 1.67^{\mathrm{bc}}$ & $10.00 \pm 0.00^{c}$ & $5.00 \pm 0.00^{c}$ & $13.33 \pm 3.33^{c}$ & $6.67 \pm 1.67^{c}$ \\
\hline 25 & $10.00 \pm 0.00^{\mathrm{b}}$ & $3.33 \pm 1.67^{\mathrm{a}}$ & $20.00 \pm 5.77^{a}$ & $6.67 \pm 1.67^{\mathrm{ab}}$ & $23.33 \pm 3.33^{b}$ & $10.00 \pm 0.00^{\mathrm{b}}$ & $40.00 \pm 0.00^{\mathrm{b}}$ & $15.00 \pm 2.89^{\mathrm{b}}$ \\
\hline 50 & $20.00 \pm 5.77^{a}$ & $3.33 \pm 1.67^{\mathrm{a}}$ & $30.00 \pm 5.77^{a}$ & $10.00 \pm 2.89^{a}$ & $50.00 \pm 5.77^{a}$ & $18.33 \pm 1.67^{\mathrm{a}}$ & $60.00 \pm 5.77^{a}$ & $25.00 \pm 2.89^{\circ}$ \\
\hline \multirow[t]{2}{*}{ CK } & $0.00 \pm 0.00^{\mathrm{b}}$ & $0.00 \pm 0.00^{\mathrm{a}}$ & $0.00 \pm 0.00^{\mathrm{b}}$ & $0.00 \pm 0.00^{c}$ & $3.33 \pm 3.33^{c}$ & $1.67 \pm 1.67^{\mathrm{d}}$ & $3.33 \pm 3.33^{c}$ & $3.33 \pm 1.67^{c}$ \\
\hline & Statics summary & & & & & & & \\
\hline S.S & 866.6 & 40.00 & 2106.6 & 226.6 & 4960.0 & 656.6 & 8000 & 976.6 \\
\hline $\mathrm{df}$ & 4 & 4 & 4 & 4 & 4 & 4 & 4 & 4 \\
\hline M.S & 216.6 & 10.00 & 526.67 & 56.67 & 1240.0 & 164.1 & 2000.0 & 244.1 \\
\hline $\mathrm{F}$ & $8.13^{* *}$ & $3.00^{*}$ & $11.29^{* *}$ & $6.80^{* * *}$ & $37.20^{* * * *}$ & $49.25^{* * *}$ & $60.00^{* * *}$ & $13.32^{* * *}$ \\
\hline
\end{tabular}

Values are described as mean \pm standard error followed by different superscripts were significantly different according to (Duncan multiple range test DMRT $p>0.05)$. S.S (Sum of square); df (Degree of freedom); M.S (Mean square); F (Significance); CK (Check); *** (Highly significant); ** (Highly significant); * (Significant). 
Probit analysis exposed the $\mathrm{LC}_{50}$, slope value, Chi-square and fiducial limits at 95\% confidence interval. Lowest $\mathrm{LC}_{50}$ values recorded by spinasterol, 22,23-dihydrospinasterol were 32.36 and $44.79 \mu \mathrm{g} / \mathrm{mL}$ after $72 \mathrm{~h}$ and $48 \mathrm{~h}$ of exposure period, respectively via contact assay. In contrast, at the same exposure period, $\mathrm{LC}_{50}$ values by residual assay were 44.57 and $58.38 \mu \mathrm{g} / \mathrm{mL}$, respectively. On the other hand, $\mathrm{LC}_{50}$ values recorded by greenhouse assay were 37.50 and $48.90 \mu \mathrm{g} / \mathrm{mL}$ at 72 and $48 \mathrm{~h}$ exposure, respectively (Table 5). Similarly, LC $_{50}$ values recorded via fernenol were 47.99 and $63.08 \mu \mathrm{g} / \mathrm{mL} ; 57.46$ and $104.46 \mu \mathrm{g} / \mathrm{mL} ; 58.67$ and $76.52 \mu \mathrm{g} / \mathrm{mL}$ at 72 and $48 \mathrm{~h}$ by contact, residual and greenhouse assay, respectively (Table 6).

Table 5. Probit analysis of the effects of spinasterol, 22,23-dihydrospinasterol on Brevicoryne brassicae.

\begin{tabular}{|c|c|c|c|c|c|c|}
\hline \multirow{2}{*}{ Bioassay } & \multirow{2}{*}{ Time (h) } & \multirow{2}{*}{$\mathrm{LC}_{50}(\mu \mathrm{g} / \mathrm{mL})$} & \multicolumn{2}{|c|}{ 95\% F.L } & \multirow{2}{*}{ Slope \pm SE } & \multirow{2}{*}{$\chi^{(2)}$} \\
\hline & & & Lower & Upper & & \\
\hline \multirow{4}{*}{ Contact } & 12 & 87.07 & 52.59 & 437.6 & $2.039 \pm 0.60$ & 1.75 \\
\hline & 24 & 61.04 & 41.42 & 151.1 & $2.089 \pm 0.52$ & 1.06 \\
\hline & 48 & 44.79 & 32.68 & 82.15 & $2.478 \pm 0.72$ & 0.31 \\
\hline & 72 & 32.36 & 23.66 & 48.39 & $2.322 \pm 0.54$ & 0.12 \\
\hline \multirow{4}{*}{ Residual } & 12 & 143.5 & 69.10 & 1634 & $1.272 \pm 1.01$ & 0.77 \\
\hline & 24 & 104.1 & 57.74 & 1003 & $1.927 \pm 0.62$ & 1.24 \\
\hline & 48 & 58.38 & 39.46 & 143.7 & $1.981 \pm 0.49$ & 0.49 \\
\hline & 72 & 44.57 & 30.99 & 90.95 & $2.078 \pm 0.59$ & 0.42 \\
\hline \multirow{4}{*}{ G. house } & 12 & 133.4 & 65.40 & 6044 & $1.870 \pm 0.68$ & 0.33 \\
\hline & 24 & 83.23 & 50.25 & 387.1 & $1.920 \pm 0.55$ & 1.09 \\
\hline & 48 & 48.90 & 36.29 & 86.10 & $2.462 \pm 0.54$ & 0.78 \\
\hline & 72 & 37.50 & 27.86 & 57.92 & $2.502 \pm 0.63$ & 0.71 \\
\hline
\end{tabular}

F.L; Fiducial limits. $\chi^{(2)}$; Chi-square.

Table 6. Probit analysis of the effects of fernenol on Brevicoryne brassicae.

\begin{tabular}{|c|c|c|c|c|c|c|}
\hline \multirow{2}{*}{ Bioassay } & \multirow{2}{*}{ Time (h) } & \multirow{2}{*}{$\mathrm{LC}_{50}(\mu \mathrm{g} / \mathrm{mL})$} & \multicolumn{2}{|c|}{ 95\% F.L } & \multirow{2}{*}{ Slope \pm SE } & \multirow{2}{*}{$x^{(2)}$} \\
\hline & & & Lower & Upper & & \\
\hline \multirow{4}{*}{ Contact } & 12 & 345.2 & 89.21 & 1145 & $1.89 \pm 0.73$ & 1.62 \\
\hline & 24 & 135.0 & 61.44 & 6531 & $0.91 \pm 1.42$ & 1.65 \\
\hline & 48 & 63.08 & 40.63 & 296.3 & $1.47 \pm 1.02$ & 0.39 \\
\hline & 72 & 47.99 & 32.59 & 94.62 & $1.27 \pm 1.11$ & 0.66 \\
\hline \multirow{4}{*}{ Residual } & 12 & 775.9 & 79.10 & 1634 & $1.43 \pm 1.19$ & 0.82 \\
\hline & 24 & 109.7 & 60.35 & 1456 & $2.05 \pm 0.69$ & 0.24 \\
\hline & 48 & 104.4 & 57.08 & 1031 & $1.82 \pm 0.58$ & 0.71 \\
\hline & 72 & 57.46 & 44.78 & 221.1 & $3.12 \pm 1.19$ & 0.24 \\
\hline \multirow{4}{*}{ G. house } & 12 & 345.2 & 98.43 & 1316 & $1.61 \pm 0.94$ & 0.61 \\
\hline & 24 & 158.0 & 67.56 & 2353 & $1.52 \pm 0.57$ & 0.99 \\
\hline & 48 & 76.72 & 49.39 & 1781 & $2.33 \pm 0.95$ & 2.39 \\
\hline & 72 & 58.67 & 38.68 & 282.9 & $2.13 \pm 0.89$ & 0.22 \\
\hline
\end{tabular}

F.L; Fiducial limits. $\chi^{(2)}$; Chi-square.

\section{Discussion}

Because of the problems associated with the use of chemical pesticides for pest administration, the introduction of natural products particularly from plants source is pressing. Essential oils or extracts of plant origin are commonly used for plant protection measures because of their effectiveness against different life stages of insect pests. However, screening of suitable candidate plants for isolation, purification and identification of active ingredients is very crucial [27]. This technique was adopted to 
obtain active compounds from C. colocynthis leaves. Additionally, different techniques are used in the extraction, separation and purification of bioactive compounds from natural plant resources.

In our results for the preliminary toxicity evaluations of the solvents extract showed significant mortality of B. brassicae. Among the solvents extracts, methanol extract afforded high mortality which is in accordance with [28-30] who reported maximum mortality of Aphis craccivora by methanolic extract of $C$. colocynthis followed by ethyl acetate and petroleum ether extract. However, the activity of the crude extract can be attributed to the existence of specific chemical compounds of the plants like fatty acids (linoleic and oleic acid) glycosides (flavonoids, phenols, saponins), terpenoids and alkaloids, etc. [31]. Recently, Ahmed et al. [32] reported that solvents (methanol, ethanol, ethyl acetate, chloroform, acetone and hexane) extract of $C$. colocynthis leaves exhibited important phytochemicals such as alkaloids, glycosides, steroids, saponins, phenol, tannins and flavonoids along with potential antioxidant activities however, acetone and ethanol extract displayed as potent antioxidants. Further, these solvents extract also exhibited pronounced insecticidal activities [33]. Butanol fraction of the methanol extract from the C. colocynthis plant possessed insecticidal properties because of the presence of flavone glucosides and two cucurbitacins glucosides [19] Moreover, Yoshikawa et al. [34] described that alcoholic extract obtained from $C$. colocynthis fruits contains various compounds comprising cucurbitacins E 2-O-a-D-glucopyranoside, colocynthosides A and B, aglycon and cucurbitacins E. Among the isolated two triterpens glycosides and four cucurbitacins from C. colocynthis leaves, one was found effective against colon cancer cells HT29 and Caco-2 [35]. Plants possess a wide range of biologic compounds involved in their mechanism of chemical defense. These natural products contain significant prospective against variety of insects [36,37]. Activity of some of the natural compounds was evaluated against Blattellea germanica which showed $\mathrm{LC}_{50}$ values as $0.07 \mathrm{mg} \mathrm{cm}^{-1}$, $0.06 \mathrm{mg} \mathrm{cm}^{-1}$ and $0.07 \mathrm{mg} \mathrm{cm}^{-1}$ via camphor, pulegone and verbenone, respectively [38]. Similarly, eugenol, isoeugenol, carvecol, thymol and p-cymene had shown anti-adulticidal activity at $1 \mathrm{mg}$ adult $^{-1}$ against $B$. germanica [39].

As different techniques are employed in the extraction, separation, purification and identification of bioactive compounds thus, following these techniques, two compounds were identified as; a mixture of spinasterol and 22,23-dihydrospinasterol and other pure compound fernenol (d:c-friedo-b':a'-neogammacer-9(11)-en-3alpha-olfern-9(11)-en-3alpha-o). Spinasterol is contained by Cucurbitaceae, Stegnospermaceae, Phytolaccaceae and Polygalaceae. In a study Meneses-Sagrero et al. [40] identified spinasterol form the methanol extract of Stegnosperma halimifolium (B.) and evaluated against cancer cell line. It was reported that spinasterol exhibited potential activity as antiproliferative against two cell lines of cervical cancer such as HeLa and RAW 264.7. Studies have also demonstrated that spinasterol exhibited different biologic activities including antidepressant [41], anti-ulcerogenic [42], anti-inflammatory [43], and antiproliferative activities [44]. Spinasterol, 22,23-dihydrospinasterol possess pharmacological and cytotoxic exertions likewise, it was isolated from Bougainvillea spectabillis and exhibited strong inhibition of xanthine oxidase being $\mathrm{IC}_{50}$ of $39.21 \mu \mathrm{M}[45]$.

Fernenol belongs to the class of organic compounds known as triterpenoids containing six isoprene units. Xian-xue $\mathrm{W}$ et al. [46] isolated this compound from the whole plant extract of Arenaria polytrichoides, however, its activity was not evaluated against pests. Similarly, Li et al. [47] isolated three terpenoids including fernenol from Ainsliaea yunnanensis showed cytotoxic effects on human acute monocytic Leukemia cell line with $\mathrm{IC}_{50}$ being $1.73 \mu \mathrm{M}$. Some studies revealed that fernenol play an important role in the inhibition of mycelial development of Colletotrichum gloeosporioides being $47.5 \mathrm{mg} \mathrm{mL}^{-1} \mathrm{EC}_{50}$, and also significantly effective against anthracnose of mango when used at $100 \mathrm{mg}$ $\mathrm{L}^{-1}$ and $200 \mathrm{mg} \mathrm{L}^{-1}$ concentration [48].

Our results demonstrated that spinasterol, 22,23-dihydrospinasterol exhibited aphicidal activity which caused significant mortality of this pest via different bioassays whereas, fernenol exhibited moderate aphicidal activity. Similar findings on extraction, purification and activity of purified compound 2-O- $\beta$-D-glucapyranosylcucurbitacin E was evaluated by Torkey et al. [49] against Aphis 
craccivora showed significant mortality with $\mathrm{LC}_{50}$ being $11,003 \mathrm{ppm}$. Moreover, aphicidal activity of Eupatorium adenophorum isolated compound 9-oxo-10, 11-dehydroageraphorone was evaluated against Pseudoregma bambucicola displayed $73.33 \%$ mortality at $2 \mathrm{mg} \mathrm{mL}^{-1}$ with $6 \mathrm{~h}$ exposure. Similarly, Nong et al. [50] recorded complete control of this pest at the same concentration after 30 days in a field experiment.

Thus, this unique and innovative research was performed first time for isolation, purification and characterization of bioactive compounds from $C$. colocynthis leaves and to investigate their insecticidal potential against cabbage aphid B. brassicae, which is an injurious pest of cabbage and other crops. It is worth mention that these compounds were isolated and evaluated from C. colocynthis for the first time against this pest.

\section{Material and Methods}

\subsection{Collection of Plants and Aphids}

C. colocynthis (Colocynth), locally recognized as Tumba, was the study plant. Leaves were collected from natural habitat of desert one climate of District Bahawalnagar, Pakistan with latitudinal and longitudinal gradients $\left(29^{\circ} 59^{\prime} 34^{\prime \prime} \mathrm{N}, 73^{\circ} 15^{\prime} 13^{\prime \prime} \mathrm{E}\right)$ from March to April 2018. This area contains dry climate with an average precipitation of $204 \mathrm{~mm}$ annually and temperature ranges from $12.7-45^{\circ} \mathrm{C}$. The samples were authenticated at Entomological Research Institute Faisalabad, Pakistan.

Cabbage aphids were collected from wild cabbage plants from the surrounding field area of Shenyang Agricultural University, Shenyang China. During the collection of aphids it was insured that no pesticides was applied on the plants. The population of the aphids was maintained on cabbage plants grown in the greenhouse at $20 \pm 5{ }^{\circ} \mathrm{C}$ and $45 \% \pm 5 \%$ relative humidity (RH), along with a photoperiod of 16:8 (light: dark).

\subsection{Extraction of Plant Material}

Extraction was performed thrice at room temperature for three days by using the cold extraction/solvent extraction method. Different organic solvents were used for extraction purposes bearing varying polarity. Then, filter the contents and volume was reduced by concentrating the filtered material through the rotary evaporator (Model R-210, Buchi Switzerland, Flawil, Switzerland). Next, the filtrate was allowed to dry for $12 \mathrm{~h}$ in a fume hood at $28^{\circ} \mathrm{C}$ and then, the dry extract was preserved in glass bottles at $4{ }^{\circ} \mathrm{C}$ for further experimentation.

\subsection{Sample Preparations and Preliminary Toxicity Evaluations}

Each of the extract obtained by using different solvents was evaluated against $B$. brassicae at $50 \mu \mathrm{g} / \mathrm{mL}$ concentration at an exposure of $6,12,24,48,72$ and $96 \mathrm{~h}$. For toxicity tests, dried extracts were dissolved in acetone ( $1 \mathrm{~mL}$ for each solvent extract) and mixed with $1 \%$ Tween-20 (prepared in distilled water) for preparing concentration. For control treatment, check (CK) was also prepared in $1 \%$ Tween-20 including acetone but excluding extract to prepare a $50 \mu \mathrm{g} / \mathrm{mL}$ concentration. Thus, to obtain homogenous mixture for separation and purification purposes, a 2-g sample from each solvent extract was taken in porcelain dish (14 g in total), dissolved in appropriate amount of dichloromethane and then added equal quantity of silica gel into it. The resulting mixture was left for overnight in a fume hood at $28^{\circ} \mathrm{C}$ to evaporate the solvent and on drying; the residue was ground to fine powder by mortar and pestle and stored in glass stopper vials at $4{ }^{\circ} \mathrm{C}$ for further use.

\subsection{Separation and Purification of Extract}

The prepared sample was separated and purified by column chromatographic techniques. The dried sample was chromatographed on silica gel column (200-300 mesh). Gradient eluent of $300 \mathrm{~mL}$ each of the eluted fraction was collected using a gradient ratio of petroleum ether and ethyl acetate. The mobile phases used for silica gel column were PE:EA of 100:0, 100:2, 100:4, 100:8, 100:16, 100:32, 
100:64, 100:100 and at the end methanol was used and finally, total 49 fractions were collected. Lastly, eight sub-fractions $(\mathrm{A}-\mathrm{H})$ were obtained followed by the mixing of the same polarity fractions. The mobile phases used for gel column Sephadex, LH-20 (40-120 $\mu \mathrm{m})$ were methanol and dichloromethane with 1:1 ratio. For further purification preparative TLC plates were used and target compounds were scrapped, dissolved in ethyl acetate and concentrated on a rotary evaporator (Buchi Switzerland R-210, Flawil, Switzerland) for obtaining pure compounds with weight calculation.

\subsection{Mass Spectrum and Elemental Analysis}

Mass spectrum data were calculated on the Triple Quad GC-MS 7000C and Triple Quad LC/MS 6440 mass Spectrometer (Agilent Technologies, Santa Clara, CA, USA). Vario EL III element analyzer was used for elemental analysis (Elementar Analyssensysteme GmbH, Frankfurt, Germany).

\subsection{Nuclear Magnetic Resonance (NMR) ${ }^{1} H-N M R$ and ${ }^{13} C-N M R$ Spectrum}

To determine ${ }^{1} \mathrm{H}-\mathrm{NMR}$ spectra, Deuterated chloroform $\left(\mathrm{CDCL}_{3}\right)$ was used as a solvent while for an internal standard, tetramethylsilane (TMS) was used. ${ }^{1} \mathrm{H}-\mathrm{NMR}$ spectra were evaluated on $300 \mathrm{MHz}$ and $600 \mathrm{MHz}$ spectrometer whereas, ${ }^{13} \mathrm{C}-\mathrm{NMR}$ spectra were assessed on a 151-MHz spectrometer (Bruker, Karlsruhe, Germany). Chemical shift values were recorded in parts per million $(\delta, \mathrm{ppm})$. The coupling constants value $(\mathrm{J})$ was described in $\mathrm{Hz}$. The splitting patterns of proton signals were also designated as follows: singlet (s), doublet (d), a doublet of doublets (dd), a doublet of the doublet of doublets (ddd), triplet $(\mathrm{t})$, the quartet (q) and the multiplet $(\mathrm{m})$

\subsection{Bioassay Study}

Activity of the compound against B. brassicae was evaluated In vitro and In vivo. For In vitro assay, contact and residual toxicity methods were adopted while; In vivo experiment was conducted under greenhouse condition of Shenyang Agricultural University. Serial concentrations such as 6.25, 12.50, 25 and $50 \mu \mathrm{g} / \mathrm{mL}$ were prepared in Tween-20 (1\%) solutions and replicated thrice.

\subsubsection{In Vitro Contact Toxicity}

For contact assay, 10 adult wingless aphids were dipped in respective concentrations for $5 \mathrm{~s}$ and released on freshly cut cabbage leaves placed in glass petri dishes. Check (CK) was prepared in $1 \%$ Tween-20 solution, but without adding a purified compound. All of the prepared petri dishes including control were placed in an incubator at $20 \pm 5{ }^{\circ} \mathrm{C}, 65 \% \mathrm{RH}$ for three days along with 16:8 (light: dark) photoperiod.

\subsubsection{In Vitro Residual Toxicity}

Fresh cabbage leaves were cut off and dipped for $10 \mathrm{~s}$ in respective concentration and dried in air for half an hour. Next, 10 adult wingless aphids were released on these leaves contained in glass petri dishes. Check (CK) was prepared in $1 \%$ Tween- 20 solution, but without adding purified compound. All of the prepared petri dishes including check were placed in an incubator at $20 \pm 5^{\circ} \mathrm{C}, 65 \% \mathrm{RH}$ for three days along with 16:8 (light: dark) photoperiod.

\subsubsection{In Vivo Toxicity}

Cabbage plants were grown and maintained in the greenhouse for the rearing of aphids and bioassay study. Prior to experiment, plants were sprayed with water to remove impurities and left for half an hour to dry in the open environment. Then, 10 wingless adult aphids were released on cabbage plants at 6-8 true leaf stage. After one hour of releasing aphids, plants were sprayed with respective concentrations (1-2 showers) with hand pump/sprayer. A control treatment was sprayed with distilled water solution in $1 \%$ Tween-20. Treated plants along with control were left for three days in greenhouse conditions. 


\subsection{Data Collection and Statistical Analysis}

Data on mortality at a specific time period of $12,24,48$ and $72 \mathrm{~h}$ were recorded regularly by examination under the microscope. Those individuals who presented no response on probing by needle were recorded as dead.

Analysis of the mortality data were performed by using analysis of variance (ANOVA). The mean mortality difference among treatments was intended at $p=0.05$ by Duncan multiple range test DMRT with IBM-SPSS statistics 25.0 version software. Probit analysis was performed using EPA Probit analysis program version 1.5 .

\section{Conclusions}

The present investigations indicated that $C$. colocynthis possess potential botanical agents. Results also demonstrated that B. brassicae showed reasonable sensitivity to isolated spinasterol, 22,23-dihydrospinasterol via all bioassay. In contrast, fernenol displayed moderate toxicity against this pest. Additionally, the contact bioassay produced higher mortality than the residual bioassay. Therefore, these chemical compounds may be introduced as alternatives to synthetic chemical insecticides. However, additional research is necessary for the purification and characterization of more bioactive constituents and their appraisals against $B$. brassicae and other field crops insect pests.

Author Contributions: Formal analysis, M.A., R.A. and H.G.; methodology, M.A; project administration, M.J.; software, J.S.; supervision, M.J.; Visualization, P.Q.; Writing—original draft, M.A.; Writing—review \& editing, M.J., P.Q. and J.S. All authors have read and agreed to the published version of the manuscript.

Funding: The financial support provided by the National Key Research \& Development Program of China (2016YFD0200500) is greatly acknowledged.

Conflicts of Interest: The authors have declared no conflict of interest.

\section{References}

1. CABI. Crop Protection Compendium. Available online: http://www.cabi.org/cpc (accessed on 3 March 2020).

2. Munthali, D. Evaluation of cabbage varieties for resistance to the cabbage aphid. Afr. Entomol. 2009, $17,1-7$. [CrossRef]

3. Swiader, J.M.; Ware, G.W.; McCollum, J.P. Producing Vegetable Crops; Interstate Printers and Publishers Inc.: Danville, IL, USA, 1992.

4. Parugrug, M.L.; Roxas, A.C. Insecticidal action of five plants against maize weevil, Sitophilus zeamais Motsch.(Coleoptera: Curculionidae). Curr. Appl. Sci. Technol. 2008, 8, $24-38$.

5. Bhatia, R.; Verma, A. Seasonal incidence of major insect pests associated with winter crop of cabbage in Himachal Pradesh. Ann. Agric. Res. 1995, 16, 278-281.

6. Opfer, P.; McGranth, D. Oregon Vegetables, Cabbage Aphid and Green Peach Aphid; Oregon State University: Corvallis, OR, USA, 2013.

7. Rashid, T.; Chen, J.; McLeod, P. Toxicity of newly isolated piperideine alkaloids from the red imported fire ant, Solenopsis invicta Buren, against the green peach aphid, Myzus persicae (Sulzer). Adv. Entomol. 2013, 2013. [CrossRef]

8. Mvumi, C.; Marais, D.; Ngadze, E.; du Toit, E.S.; Tsindi, A. Effect of moringa extract on the leaf anatomy and yield potential of tomato infected by Alternaria solani. South Afr. J. Plant Soil 2018, 35, 389-392. [CrossRef]

9. Springob, K.; Kutchan, T.M. Introduction to the different classes of natural products. In Plant-Derived Natural Products; Donald Danforth Plant Science Center: St. Louis, MO, USA, 2009; pp. 3-50.

10. Fukuta, M.; Dang Xuan, T.; Deba, F.; Tawata, S.; Dang Khanh, T.; Min Chung, I. Comparative efficacies in vitro of antibacterial, fungicidal, antioxidant, and herbicidal activities of momilatones A and B. J. Plant Interact. 2007, 2, 245-251. [CrossRef]

11. Soam, P.S.; Singh, T.; Vijayvergia, R. Citrullus colocynthis (LINN.) and Luffa acutangula (L.) roxb, schrad. source of bioinsecticides and their contribution in managing climate change. IJABPT 2013, 4, 7-9.

12. Memon, U.; Brohi, A.; Ahmed, S.W.; Azhar, I.; Bano, H. Antibacterial screening of Citrullus colocynthis. Pak. J. Pharm. Sci. 2003, 16, 1-6. 
13. Mohammad, D.; Al-Khateeb, M.; Riyadh, E.; Al-Hashem, F.; Nabil, B.; Mohammad, K. In vivo, acute, normo-hypoglycemic, antihyperglycemic, insulinotropic actions of orally administered ethanol extract of Citrullus colocynthis (L.) Schrab pulp. Am. J. Biochem. Biotechnol. 2009, 5, 119-126.

14. Kumar, S.; Kumar, D.; Jusha, M.; Saroha, K.; Singh, N.; Vashishta, B. Antioxidant and free radical scavenging potential of Citrullus colocynthis (L.) Schrad. methanolic fruit extract. Acta Pharm. 2008, 58, 215-220. [CrossRef]

15. Dehghani, F.; Panjeh, S.M.; Talaei, K.T.; Mesbah, A.S.; Azizi, M. Toxic effects of hydroalcoholic extract of Citrullus colocynthis on pregnant mice. Iran. J. Vet. Res. Shiraz Univ. 2008, 9, 42-45.

16. Adam, S.; Al-Yahya, M.; Al-Farhan, A. Response of Najdi sheep to oral administration of Citrullus colocynthis fruits, Nerium oleander leaves or their mixture. Small Rumin. Res. 2001, 40, 239-244. [CrossRef]

17. Sturm, S.; Schneider, P.; Seger, C.; Stuppner, H. Analysis of Citrullus colocynthis cucurbitacine derivatives with HPLC-SPE-NMR. Sci. Pharm. 2009, 77, 254. [CrossRef]

18. Seger, C.; Sturm, S.; Mair, M.E.; Ellmerer, E.P.; Stuppner, H. 1H and 13C NMR signal assignment of cucurbitacin derivatives from Citrullus colocynthis (L.) Schrader and Ecballium elaterium L.(Cucurbitaceae). Magn. Reson. Chem. 2005, 43, 489-491. [CrossRef] [PubMed]

19. Delazar, A.; Gibbons, S.; Kosari, A.R.; Nazemiyeh, H.; Modarresi, M.; Nahar, L.; Sarker, S.D. Flavone C-glycosides and cucurbitacin glycosides from Citrullus colocynthis. DARU J. Pharm. Sci. 2006, 14, 109-114.

20. Maatooq, G.T.; El-Sharkawy, S.H.; Afifi, M.; Rosazza, J.P. Cp-hydroxybenzoylglycoflavones from Citrullus colocynthis. Phytochemistry 1997, 44, 187-190. [CrossRef]

21. Jayaprakasam, B.; Seeram, N.P.; Nair, M.G. Anticancer and antiinflammatory activities of cucurbitacins from Cucurbita andreana. Cancer Lett. 2003, 189, 11-16. [CrossRef]

22. Song, F.; Dai, B.; Zhang, H.-Y.; Xie, J.-W.; Gu, C.-Z.; Zhang, J. Two new cucurbitane-type triterpenoid saponins isolated from ethyl acetate extract of Citrullus colocynthis fruit. J. Asian Nat. Prod. Res. 2015, 17, 813-818. [CrossRef]

23. Ding, L.; Chen, Y.; Wu, F. Constituents of the root of Berneuxia thibetica Decne. Zhongguo Zhong Yao Za Zhi= Zhongguo Zhongyao Zazhi= China J. Chin. Mater. Med. 1991, 16, 289-290, 318.

24. Sinha, B.; Sasmal, D.; Basu, S. Pharmacological studies on Melothria maderaspatana. Fitoter (Milano) 1997, 68, $75-78$.

25. Glasby, J.S. Directory of Plants Containing Secondary Metabolites; Taylor \& Francis group CRC Press: Boca Raton, FL, USA, 1991.

26. Rai, P.; Paudel, N.; Shakya, S. Cytological Effects of Leaf Extract of Artemisia vulgaris L. on Meristematic Cells of Allium cepa L. Our Nat. 2012, 10, 242-248. [CrossRef]

27. Chermenskaya, T.D.; Stepanycheva, E.A.; Shchenikova, A.V.; Savelieva, E.I.; Chakaeva, A.S. Insecticidal effects of Ungernia severtzovii bulb extracts against the grain aphid Schizaphis graminum (Rondani). Ind. Crop. Prod. 2012, 36, 122-126. [CrossRef]

28. Shehawy, A.A.; Khalil, A.-H.E.; Maklad, A.M.; Qari, S.H. Toxicity Effects and Biochemical Changes of Insecticide Alternatives on Cowpea Aphid,(Aphis craccivora)(Homoptera: Aphididae). Egypt. Acad. J. Biol. Sci. F. Toxicol. Pest Control 2019, 11, 129-138. [CrossRef]

29. Shehawy, A.; Alshehri, A. Toxicity and biochemical efficacy of Novel pesti-cides against Aphis craccivora (Hemiptera: Aphididae) in relation to enzymatic activity. J. Plant Prot. Path. Mansoura Univ. 2015, 6, 1507-1516.

30. Ibrahim, H.Y.E. Characterization of chemical constituents of Citrullus colocynthis (L.) extracts and their relation to toxicity against cowpea aphid, aphis craccivora koch. JPPP 2015, 6, 1323-1336. [CrossRef]

31. Yaniv, Z.; Shabelsky, E.; Schafferman, D. Colocynth: Potential arid land oilseed from an ancient cucurbit. Perspect. New Crop. New Uses 1999, 3, 257-261.

32. Ahmed, M.; Ji, M.; Peiwen, Q.; Liu, Y.; Gu, Z.; Sikandar, A.; Iqbal, M.; Javeed, A. Phytochemical screening, total phenolics and flavonoids content and antilxidant activities of Citrullus colocynthis L. and Cannabis sativa L. Appl. Ecol. Environ. Res. 2019, 17, 6961-6979. [CrossRef]

33. Ahmed, M.; Peiwen, Q.; Gu, Z.; Liu, Y.; Sikandar, A.; Hussain, D.; Javeed, A.; Shafi, J.; Iqbal, M.F.; An, R. Insecticidal activity and biochemical composition of Citrullus colocynthis, Cannabis indica and Artemisia argyi extracts against cabbage aphid (Brevicoryne brassicae L.). Sci. Rep. 2020, 10, 1-10. [CrossRef] 
34. Yoshikawa, M.; Morikawa, T.; Kobayashi, H.; Nakamura, A.; Matsuhira, K.; Nakamura, S.; Matsuda, H. Bioactive saponins and glycosides. XXVII. Structures of new cucurbitane-type triterpene glycosides and antiallergic constituents from Citrullus colocynthis. Chem. Pharm. Bull. 2007, 55, 428-434. [CrossRef]

35. Chawech, R.; Jarraya, R.; Girardi, C.; Vansteelandt, M.; Marti, G.; Nasri, I.; Racaud-Sultan, C.; Fabre, N. Cucurbitacins from the leaves of Citrullus colocynthis (L.) Schrad. Molecules 2015, 20, 18001-18015. [CrossRef]

36. Michaelakis, A.; Papachristos, D.; Kimbaris, A.; Koliopoulos, G.; Giatropoulos, A.; Polissiou, M.G. Citrus essential oils and four enantiomeric pinenes against Culex pipiens (Diptera: Culicidae). Parasitol. Res. 2009, 105, 769. [CrossRef] [PubMed]

37. Lardeux, F.; Depickère, S.; Duchon, S.; Chavez, T. Insecticide resistance of Triatoma infestans (Hemiptera, Reduviidae) vector of Chagas disease in Bolivia. Trop. Med. Int. Health 2010, 15, 1037-1048. [CrossRef]

38. Jung, W.-C.; Jang, Y.-S.; Hieu, T.T.; Lee, C.-K.; Ahn, Y.-J. Toxicity of Myristica fragrans seed compounds against Blattella germanica (Dictyoptera: Blattellidae). J. Med. Entomol. 2007, 44, 524-529. [CrossRef]

39. Yeom, H.-J.; Kang, J.S.; Kim, G.-H.; Park, I.-K. Insecticidal and acetylcholine esterase inhibition activity of Apiaceae plant essential oils and their constituents against adults of German cockroach (Blattella germanica). J. Agric. Food Chem. 2012, 60, 7194-7203. [CrossRef] [PubMed]

40. Meneses-Sagrero, S.E.; Navarro-Navarro, M.; Ruiz-Bustos, E.; Del-Toro-Sánchez, C.L.; Jiménez-Estrada, M.; Robles-Zepeda, R.E. Antiproliferative activity of spinasterol isolated of Stegnosperma halimifolium (Benth, 1844). Saudi Pharm. J. 2017, 25, 1137-1143. [CrossRef] [PubMed]

41. Socała, K.; Wlaź, P. Evaluation of the antidepressant-and anxiolytic-like activity of $\alpha$-spinasterol, a plant derivative with TRPV1 antagonistic effects, in mice. Behav. Brain Res. 2016, 303, 19-25. [CrossRef]

42. Klein, L.C.; Gandolfi, R.B.; Santin, J.R.; Lemos, M.; Cechinel Filho, V.; de Andrade, S.F. Antiulcerogenic activity of extract, fractions, and some compounds obtained from Polygala cyparissias St. Hillaire \& Moquin (Polygalaceae). Naunyn-Schmiedeberg's Arch. Pharmacol. 2010, 381, 121-126.

43. Borges, F.R.; Silva, M.D.; Córdova, M.M.; Schambach, T.R.; Pizzolatti, M.G.; Santos, A.R. Anti-inflammatory action of hydroalcoholic extract, dichloromethane fraction and steroid $\alpha$-spinasterol from Polygala sabulosa in LPS-induced peritonitis in mice. J. Ethnopharmacol. 2014, 151, 144-150. [CrossRef]

44. Ntie-Kang, F.; Yong, J.N. The chemistry and biological activities of natural products from Northern African plant families: From Aloaceae to Cupressaceae. RSC Adv. 2014, 4, 61975-61991. [CrossRef]

45. Chang, W.; Chang, Y.; Lu, F.; Chiang, H.-C. Inhibitory effects of phenolics on xanthine oxidase. Anticancer Res. 1994, 14, 501-506.

46. Wu, X.X.; Li, M.; Zhang, Q.; Cheng, L. Chemical constituents from Arenaria polytrichoides (II). Chem. Res. Appl. 2013, 25, 333-335.

47. Li, J.; Zhang, B.; Liu, H.; Zhang, X.; Shang, X.; Zhao, C. Triterpenoids from Ainsliaea yunnanensis Franch. and their biological activities. Molecules 2016, 21, 1481. [CrossRef] [PubMed]

48. Yenjit, P.; Issarakraisila, M.; Intana, W.; Chantrapromma, K. Fungicidal activity of compounds extracted from the pericarp of Areca catechu against Colletotrichum gloeosporioides in vitro and in mango fruit. Postharvest Biol. Technol. 2010, 55, 129-132. [CrossRef]

49. Torkey, H.; Abou-Yousef, H.; Abdel Azeiz, A.; Hoda, E. Insecticidal effect of cucurbitacin E glycoside isolated from Citrullus colocynthis against Aphis Craccivora. Aust. J. Basic Appl. Sci. 2009, 3, 4060-4066.

50. Nong, X.; Fang, C.-L.; Wang, J.-H.; Gu, X.-B.; Yang, D.-Y.; Liu, T.-F.; Fu, Y.; Zhang, R.-H.; Zheng, W.-P.; Peng, X.-R. Acaricidal activity of extract from Eupatorium adenophorum against the Psoroptes cuniculi and Sarcoptes scabiei in vitro. Vet. Parasitol. 2012, 187, 345-349. [CrossRef]

Sample Availability: Samples of the compounds are available from the authors. 